nasium zu bringen, gerecht zu werden. Die analytische Geometrie ist wesentlich umgeändert worden und nunmebr recht ausfährlich und korrekt behandelt. Stellenweise enthält das Buch mehr, als es der vorgesehriebene Lehrstoff verlangt, was wohl kaum als Nachteil ausgelegt werden kann. Im ganzen macht das Buch einen sehr gediegenen Eindruck and kann den Fachgenossen wärmstens empfohlen werden.

G. v. Alth.

\title{
Vorlesungen über theoretische und physikalische Chemie.
} Von J. H. van't Hoff. Drittes Heft, zweite Auflage (Braunschweig, 1903).

Mit dem Erscheinen dieses Heftes wird die zweite Auflage dieser wertvollen Vorlesungen abgeschlossen. Gegen die erste Auflage weist anch dieser T'eil keine größeren Änderungen auf; doch gibt sich das Bestreben des Ver. fassers, den jüngsten Forschungsergebnissen Rechnung zu tragen, durch eine große Zahl kleiner, in den Text verwebter Zusätze, durch nene Literaturangaben etc. zu erkennen. Einen recht dankenswerten Zusatz bildet das beigefügte Namen- und Sachregister, welches ein Nachschlagen wesentlich erleichtert.

J. B.

Vorlesungen über die Theorie der Determinanten. Von Leopold Kronecker, bearbeitet und fortgeführt von Dr. Kurt $\mathrm{Hensel}$. I. Band, 1. bis 21. Vorlesung. (XII. + 390, gr. 8.) Leipzig; B. G. Teubner, 1903.

In dieser Ausgabe der Vorlesungen Kroneckers über Determinanten hat sich der Verfasser die Aufgabe gestellt, unter sorgfältiger Erhaltung ihrer Grundprinzipien und unter Benützung seiner einfachen und wirksamen Methoden den Stoff so zu bearbeiten und fortzuführen, daß dieses Werk eine systematische Darstellung der modernen Determinanten und ihrer wichtigsten Anwendungen enthält.

Nach Kroneckers Auffassung bildet die Determinantentheorie einen Teil der allgemeinen Arithmetik, und zwar denjenigen, welcher sich mit den Eigenschaften der linearen Funktionen beschäftigt. Von diesem Gesichtspunkte aus pflegte er zunächst eine sehr eingehende Darstellung der Determinanten zweiter und dritter Ordnung und ihrer einfachsten Anwendungen zu geben, aber schon auf dieser Stufe die ihm eigentümlichen Begriffe der Äquivalenz linearer Systeme, deren Komposition aus elementaren Systemen und die Anwendung dieser Begriffe anf bilineare Formen und Gittersysteme zu bringen Ebenso wird schon hier der Begriff und die Bedentung des Ranges einer Determinante erklärt und benützt. Die Quaternionen werden bereits gestreift und das ganze Gebiet durch geometrische Anwendung einfacher Art belebt.

Bei diesen mehr einleitenden und vorbereitenden Untersuchungen hat nun Hensel dem Leser sorgfältig jede Schwierigkeit ans dem Wege geräumt, vielleicht zu. sorgfältig, denn es bedarf einer gewissen Überwindung, um nicht in Flüchtigkeit bei der Lektüre zu verfallen. Es ist ja hier sehr schwierig die richtige Grenze zu finden und sie wird immer subjektiv bleiben. Eine Darstellung, welche dem Leser gar nichts oder wenig zumutet hat gewiß große Vorteile, aber anderseits ist doch sicher der Gewinn, den man aus der 